\title{
Motivated attention in emotional picture processing is reflected by activity modulation in cortical attention networks
}

\author{
Stephan Moratti, ${ }^{*}$ Andreas Keil, and Margarita Stolarova \\ Department of Psychology, University of Konstanz, Constance, Germany
}

Received 8 July 2003; revised 17 October 2003; accepted 22 October 2003

In the present study, we presented high (pleasant and unpleasant)and low (neutral)-arousing emotional pictures in a steady state visual evoked field paradigm while recording the magnetoencephalogram. Applying a minimum norm estimation (MNE) technique, we determined the origin and the strength of the evoked neuromagnetic field. In addition to magnetocortical data, we examined subjective ratings, heart rate change and viewing time to obtain a multivariate data base of emotional experience related to the present paradigm. As evidenced by the MNE, pictures rated as high arousing elicited greater activity in frontoparietal cortical networks than low-arousing pictures, with a right hemispheric predominance. This effect was also observed in occipito-temporal regions but to a lesser extent. Longer viewing times for high-arousing pictures and sustained heart rate deceleration for high-arousing unpleasant pictures indicated that these stimuli were of high motivational relevance compared to neutral pictures. Taken together, we argue that activity in higher-order frontoparietal cortical attention networks is modulated by emotional arousal. In turn, this attention network influences activity in systems performing stimulus processing.

(C) 2004 Elsevier Inc. All rights reserved.

Keywords: Motivated attention; Emotion; Magnetoencephalography; Steady state visual evoked field; Attention network

\section{Introduction}

Recent developments in emotion theory have emphasized the importance of motivational systems and processes (Lang et al., 1997). As one possible laboratory model of emotional processing, viewing of emotional pictures has been shown to co-vary with measures indicative of the motivational state of the organism. For instance, longer viewing time and higher skin conductance can be observed for stimuli rated as high in emotional arousal, compared to calm picture stimuli (Lang et al., 1998). Likewise, measures of central nervous activity correlated with subjective arousal ratings of emotional pictures. Studies using Event-Related Potentials

* Corresponding author. Department of Psychology, University of Konstanz, PO Box D25, D-78457 Constance, Germany. Fax: +49-7531884601.

E-mail address: Stephan.Moratti@uni-konstanz.de (S. Moratti).

Available online on ScienceDirect (www.sciencedirect.com.)
(ERPs) as a dependent variable demonstrated that high-arousing stimuli (pleasant as well as unpleasant pictures) produce a sustained late-positive wave, which is attenuated for less-arousing neutral images (Ito et al., 1998; Mini et al., 1996; Palomba et al., 1997; Schupp et al., 2000). This late-positive wave can be maintained for several seconds until picture presentation offset and is interpreted as reflecting greater activity in systems processing different aspects of the stimulus, including their motivational relevance (Cuthbert et al., 2000). Using dense array EEG and a source estimation technique, Keil et al. (2002) identified occipital and posterior parietal regions with a right hemispheric dominance as a possible origin of the arousal-modulated latepositive wave.

In addition to these late ERP modulations by emotional intensity, several studies have reported ERP changes at earlier stages of visual processing. In a rapid serial picture presentation paradigm, Junghöfer et al. (2001) observed enhanced negativity at posterior recording sites in time ranges around $200 \mathrm{~ms}$ after onset of high-arousing stimuli compared to neutral ones. Consistent with this finding, the visual N1 component has been found to vary with affective arousal of visual stimuli (Keil et al., 2002; Pizzagalli et al., 1999).

Taken together, this evidence suggests that the affective or motivational significance of a stimulus may amplify sensory processing at early stages of visual information processing reflecting fast discrimination. Thus, motivated attention (Lang et al., 1997) may represent automatic allocation of processing resources to visual features that are motivationally significant, paralleling a neural 'sensory gain' mechanism as hypothesized by authors in the field of selective attention (Hillyard and AnlloVento, 1998). In this framework, the late-positive wave has been interpreted as a measure of cortical activity for allocating more attentional resources to arousing stimuli over time, whereas earlier amplification such as found in the range of the N1 component may indicate initial cortical facilitation for relevant features.

In addition to electrophysiological measures, hemodynamic/ metabolic imaging studies have highlighted the role of cortical facilitation in attentional processes. For instance, recent imaging research in normal and pathological states has illuminated a frontoparietal attention network that mediates orienting to external stimuli and maintaining the alert state (Fernandez-Duque and Posner, 2001). Imaging research with healthy participants not 
only revealed frontoparietal networks in visuospatial orienting (Corbetta, 1998) but also identified the superior parietal cortex as being involved in attention shifts between nonspatial dimensions such as color and shape (Le et al., 1998; Rushworth et al., 2001). Pardo et al. (1991) investigated neural activity during a visual and somatosensory sustained attention task using positron emission tomography (PET). Regardless of sensory modality, they found an increase in blood flow in right prefrontal and right superior parietal cortices. Thus, a general frontoparietal network in the right hemisphere appears to be involved in orienting to a new stimulus as well as in maintaining attention to that stimulus. These brain areas project to subcortical structures, for example, the pulvinar of the thalamus linking ventral visual systems for object identification with the attention network (Fernandez-Duque and Posner, 2001).

In research on emotion, deep structures such as the amygdaloid complex have been discussed as central areas interacting with the ventral visual system (LeDoux, 1992; Shi and Davis, 2001), suggesting some degree of differentiation between 'cognitive' and 'emotional' attention systems. Nevertheless, the location of activated brain areas reported during processing of emotional stimuli strikingly overlap with brain areas associated with orienting and sustained attention. In particular, the prefrontal cortex, the anterior cingulate, and the parietal cortex have been considered as crucial for emotional function (Davidson et al., 1999).

In the light of the data reported above, the question arises if high-arousing visual stimuli increase the activity not only in sensory cortical areas but also in cortical networks mediating attentional processes over time. This would provide additional support for the concept of 'motivated attention' in emotion. The Steady State Visual Evoked Potential (SSVEP) has proved useful in electrocortical investigations of attentional processes over time (Müller and Hillyard, 2000). It can be recorded as a continuous brain response elicited by a repetitive stimulus that is periodically modulated in intensity at a fixed rate of $6-8 \mathrm{~Hz}$ or greater. The resulting waveform is oscillatory in nature, having the same fundamental frequency as the driving stimulus, often including higher harmonics (Regan, 1989). The SSVEP is sensitive for tonic changes of the organism such as anaesthesia (Plourde and Picton, 1990), sleep (Picton et al., 1987), or vigilance (Silberstein et al., 1990). But also phasic changes in central nervous activity have been observed using the SSVEP technique such as visual spatial selective attention whereby greater SSVEP amplitudes for attended vs. unattended stimuli were found (Morgan et al., 1996; Müller et al., 1998). Because of high correlation with the visual N1 component, the amplitude enhancement for attended stimuli was considered reflecting a greater signal-to-noise ratio in sensory systems as a result of a sensory gain mechanism facilitating feature discrimination (Muller and Hubner, 2002). The neuromagnetic counterpart of the SSVEP, the Steady State Visual Evoked Field (SSVEF) measured with magnetoencephalography (MEG), can also be used to study higher-order attentional processes such as conscious perception (Srinivasan et al., 1999). Advantages of this technique are related to the fact that they enable the researcher to collect a high number of trials in short periods of time, and to use frequency-domain analyses allowing for easy extraction of meaningful dependent variables that reflect the driving frequency (e.g. Fourier-based approaches). As a conceptual advantage, widely distributed functional networks oscillating coherently at the driving frequency can be examined at high temporal resolution and signal-to-noise ratios.
Few studies (Keil et al., 2003; Kemp et al., 2002) have used SSVEPs to investigate the effect of emotional pictures on electrocortical brain activity. Kemp et al. (2002) examined the influence of pictures from the International Affective Picture System (IAPS) on SSVEPs produced by a $13 \mathrm{~Hz}$ diffuse peripheral flicker. They manipulated the valence dimension (pleasantness) and kept the arousal dimension low and constant for the valence categories (unpleasant, pleasant, neutral). These authors found a frontal latency reduction of the SSVEP for the unpleasant and pleasant picture categories compared to the neutral pictures as predicted by the model of Heller et al. (1997). Furthermore, unpleasant pictures decreased the amplitude of the flicker-induced SSVEP in frontal electrodes.

In a previous SSVEP study (Keil et al., 2003), we examined the SSVEP produced by flickering $(10 \mathrm{~Hz})$ IAPS pictures themselves. We manipulated the arousal dimension of the picture content resulting in high (pleasant and unpleasant) and low (neutral)-arousing pictures to direct motivated attention to higharousing stimuli. The SSVEP signal was most pronounced for high arousing compared to neutral pictures at occipital and parietal electrode sites. Phase information of the SSVEP suggested coactivation of frontal and temporal regions when viewing affectively arousing pictures. In general, EEG-based measures are limited with respect to spatial resolution. Neuromagnetic fields measured with MEG are not distorted by varying conductivities resulting from different tissues that affect electric field potentials. Consequently, in the case of MEG, using a homogenous sphere as a volume conductor model for source analysis introduces less spatial error. Therefore, the aim of the present study was to examine cortical sources of the SSVEF as modulated by emotional arousal, using MEG.

We hypothesized that pictures high in arousal should provoke greater SSVEF amplitudes reflecting an enhanced signal-to-noise ratio in sensory systems as a result of a sensory gain mechanism. Since the SSVEF response is a correlate of ongoing, continuous stimulation, it represents integrated stimulus-driven neural activity over time. This should lead to oscillations in neuronal networks responsible for affective picture processing at the frequency of the driving stimulus. Therefore, we expect greater SSVEF amplitude for high-arousing pictures not only in extrastriate cortex but also in higher attentional networks reflecting orienting and maintaining an alert state over a longer period. Applying the Minimum Norm Estimate (MNE; Hauk et al., 1998, 2002) to the SSVEF response to determine the sources of the neuromagnetic field, we expected enhanced source activity in higher visual, parietal, and frontal brain areas. Heart rate (HR) was recorded to validate the effectiveness of picture presentation with respect to the observers' affective physiology. Evaluative judgments and viewing time were obtained as subjective measures of stimulus valence and intensity.

\section{Methods}

\section{Participants}

Fifteen paid volunteers ( 8 females, 7 males, 12 right handed, 1 left handed and 2 ambidextrous; Oldfield, 1971) gave written consent to participate in the study. Their mean age was 25.5 years (range: 20-32 years). They had normal or corrected-to-normal vision and no family history of photic epilepsy. They did not report a recent critical live event or any history of psychotherapy. 
Stimuli

Sixty colored pictures from the IAPS were used as stimuli. According to the normative IAPS ratings (CSEA, 1999) 20 high arousing pleasant (erotic couples and happy families), 20 high arousing unpleasant (mutilated bodies and attack scenes) and 20 low arousing neutral pictures (household scenes and neutral persons) were chosen. Mean normative pleasure ratings on a 9 point scale for pleasant, neutral and unpleasant pictures were $7.33( \pm 0.13), 4.87( \pm 0.05)$ and $2.76( \pm 0.19)$, mean normative arousal ratings were $5.44( \pm 0.24), 2.8( \pm 0.13)$ and $6.9( \pm 0.07)$, respectively. Brightness, contrast and color spectra of the stimuli were adjusted such that there were no systematic differences between picture categories with respect to these parameters.

Using a video projector (JVC ${ }^{\mathrm{TM}}$, DLA-G11E) and a mirror system, the pictures were projected on a screen in the magnetically shielded MEG chamber. The pictures were displayed in a pseudo random order, subtending a visual angle of $10^{\circ}$ both horizontally and vertically. A fixation point was marked in the center of the display and was present throughout the whole experiment. In each trial, a picture was shown in a flickering mode of $10 \mathrm{~Hz}$ for a period of $6000 \mathrm{~ms}$ resulting in $60 \mathrm{on} / \mathrm{off}$ cycles, the picture being depicted for $40 \mathrm{~ms}$, followed by $60 \mathrm{~ms}$ black screen during each cycle. During the inter-trial interval, which varied randomly between 8 and 12 seconds, a black screen was shown.

\section{Procedure}

Upon arriving at the laboratory, participants were familiarized with the MEG chamber. Thereafter, they were prepared for the MEG recording. First, subjects were shown three pictures from the IAPS representing high-arousing pleasant, unpleasant, and low-arousing neutral pictures that were not part of the stimuli presented during the MEG session. After giving informed consent, participants were screened for family history of epilepsy, recent critical live events and history of psychotheraphy. Handedness was determined using the Edinburgh Inventory(Oldfield, 1971). For artifact control, four electrodes for the electrooculogram (EOG) were attached, two at the left and right outer canthus and two above and below the right eye. Additionally, two electrodes for the electrocardiogram (ECG) were placed on the left and right medial forearms. Then, subjects were seated in the MEG chamber, their head shapes were digitized, and index points (left and right periauricular points, nasion, a pseudo- $\mathrm{Cz}$ and pseudo-inion point at the forehead) were determined to calculate the relative head position within the MEG helmet for source analysis. Finally, subjects were placed under the MEG sensors.

The experimental session started with the presentation of 60 flickering $(10 \mathrm{~Hz})$ pictures in a pseudorandomized order. Randomization was restricted such that a straight sequence of three pictures from the same affective category (unpleasant, pleasant, neutral) did not occur. After the first block subjects were offered a brief pause and a second block of the same 60 pictures in a different order was run. At the end of the MEG session, subjects left the MEG chamber and electrodes were removed.

In a third block outside the MEG chamber, participants were asked to rate the pictures regarding emotional valence and arousal using the Self-Assessment Manikin (SAM) self-report scale (Lang, 1980). The same 60 pictures were presented on a computer screen in a $10-\mathrm{Hz}$ flickering mode in a different order compared to both MEG blocks. Subjects were allowed to view the pictures as long as they wanted to, but had to press a key to stop the picture presentation before doing their rating. After finishing, the evaluation of the current picture, the participant could start the presentation of the next picture by a second key press. The time interval between picture onset and offset was recorded as an index of viewing time. At the end of the experiment, participants were paid $10 €$ (approximately 10 US dollars).

\section{Data acquisition and preprocessing}

The MEG was recorded continuously, and digitized at a rate of 678.17 Hz, using a 148-channel whole head system (MAGNES ${ }^{\text {TM }}$ $2500 \mathrm{WH}, 4 \mathrm{D}$ Neuroimage, San Diego, USA). A band-pass filter of $0.1-200 \mathrm{~Hz}$ was applied on line. The EOG and ECG acquisition was done with a Synamps amplifier (NEUROSCAN ${ }^{\mathrm{TM}}$ ) using $\mathrm{Ag} / \mathrm{Cl}$ electrodes.

The MEG data were decimated by a factor of 2 and digitally band-pass filtered between 1 and $20 \mathrm{~Hz}$ (slopes: 6 and $24 \mathrm{~dB} /$ octave, respectively) before averaging for picture category (pleasant, neutral, unpleasant) over $6000 \mathrm{~ms}$ subtracting a 200-ms baseline. Eye artifacts were corrected using the algorithm implemented in BESA ${ }^{\mathrm{TM}}$ software (Berg and Scherg, 1994). Additionally, the MEG was visually inspected for movement artifacts. For each category average (baseline corrected $6000 \mathrm{~ms}$ ), mean SSVEFs were derived using a moving window averaging procedure (time window $400 \mathrm{~ms}$, time shift $100 \mathrm{~ms}$ ) to extract four cycles of the $10 \mathrm{~Hz}$ brain response from the MEG signal (Perlstein et al., 2003). These four cycles per category were submitted to source analysis.

Heart rate changes were evaluated using in-house-software written in MATLAB ${ }^{\mathrm{TM}}$. The algorithm of the software estimated the HR change over 6000 -ms picture presentation in 500-ms steps using a 2000-ms prestimulus baseline. Thereby, the frequency of the occurrence of an R-wave was derived from the interbeatinterval and weighted according to the distance from a $500-\mathrm{ms}$ step within the time vector. The HR change was transformed in beats per minutes (bpm).

For viewing time estimation, the time between the key press starting the presentation of a new picture and the key press before picture evaluation was used as a measure of viewing time to exclude evaluation time. The viewing time was recorded with an $\mathrm{IBM}^{\mathrm{TM}}$ compatible personal computer.

\section{Source analysis}

The average data resulting from the moving window averaging procedure was submitted to source analysis. Using a minimum norm estimate (MNE) (Hämäläinen and Ilmoniemi, 1994), cortical sources were estimated. This linear estimation technique assumes that the data vector $\mathbf{b}$, which contains the recorded magnetic field distribution over the sensors, can be described as the product of the lead-field matrix A, which specifies the sensor's sensitivity to the sources located, for example, on a shell, and a current density vector $\mathbf{x}$ of these sources plus a noise component $\varepsilon$ (Grave de Peralta Menendez et al., 1997):

$b=A x+\varepsilon$ 
The MNE is characterized by minimizing the power of the estimated current density of the sources by multiplying the pseudoinverse of $\mathbf{A}$ with the data $\mathbf{b}$ :

$\hat{x}^{T} \hat{x}=\min$

where $\hat{x}$ is the estimated solution, and $\hat{x}^{\mathrm{T}}$ is the transpose of $\hat{x}$. The fit of $\hat{x}$ to the data is quantified using the residual variance:

$(A \hat{x}-b)^{\mathrm{T}}(A \hat{x}-b)=\hat{\varepsilon}>0$

Given the presence of noise and the high number of sensors, a regularization is necessary to obtain a stable solution. A commonly applied regularization method is the Tikhonov-Phillips regularization during pseudoinversion of $\mathbf{A}$, leading to following solution for $\hat{x}$ :

$\hat{x}=A^{\mathrm{T}}\left(A A^{\mathrm{T}}+\lambda I\right)^{-1} b$

where $\mathbf{I}$ is the identity matrix and $\lambda$ the regularization parameter (Bertero et al., 1988). Regularization aims at suppressing the noise part of the solution. Therefore, increasing $\lambda$ should enhance the stability of the solution. Larger $\lambda$ values lead to increased residual variance $\hat{\varepsilon}$ and smoothing of the estimated solution (Bertero et al., 1988).

Plotting the norm of the solution estimate $\|\hat{x}\|^{2}$ versus the residual variance $\hat{\varepsilon}$, the regularization parameter $\lambda$ at the turning point of this curve was considered as an optimal tradeoff between a stable solution estimate and a good data fit. For the determination of $\lambda$, the solution was calculated for an average over all affective categories (unpleasant, pleasant, and neutral) for each subject. This procedure should prevent that different regularization parameters per affective category could account for condition effects. The different regularization parameters for each subject are shown in Table 1.

We used the algorithm suggested by Hauk et al. (2002) to calculate the MNE. To this end, the lead-field matrix A was computed for each participant, based on information on the center of a fitted sphere to the digitized head shape, and the positions of the MEG sensors relative to the head. A spheric shell with evenly distributed 197 dipole locations served as source space. To be able to average the obtained MNE solutions over different sub-

Table 1

Tikhonov regularization factors for each subject determined by the $L$-curve criterion

\begin{tabular}{ll}
\hline Subject & Tikhonov regularization factor $\lambda$ \\
\hline S01 & 0.017 \\
S02 & 0.017 \\
S03 & 0.021 \\
S04 & 0.027 \\
S05 & 0.011 \\
S06 & 0.017 \\
S07 & 0.023 \\
S08 & 0.017 \\
S09 & 0.013 \\
S10 & 0.023 \\
S11 & 0.011 \\
S12 & 0.013 \\
S13 & 0.011 \\
S14 & 0.015 \\
S15 & 0.013 \\
\hline
\end{tabular}

jects, we employed a shell with a fixed radius of $6 \mathrm{~cm}$ (see Fig. 6). Utilizing source spaces formed by a shell with a common radius to calculate grand average data led to solutions with adequate spatial resolution (Knosche et al., 1996). The radius of $6 \mathrm{~cm}$ was chosen as a tradeoff between depth sensitivity and spatial resolution (Hauk et al., 2002). Each of the 197 dipole locations consisted of two perpendicular dipoles oriented tangentially to the shell surface. This resulted in two MNEs for each dipole location. Computing the MNE (Eq. (4)) leads to an estimation of dipole strength for each of the two perpendicular oriented dipoles at each location resulting in an activity estimation distributed over the shell.

Using an FFT algorithm, the amplitude of the 10-Hz Fourier component of the brain response was estimated separately for each dipole orientation. The square root of the sum of squares of the amplitudes for the two orientations served as a measure of the total amplitude of the $10-\mathrm{Hz}$ Fourier component at one location:

$\mathrm{Amp}_{i}=\sqrt{\mathrm{Amp}_{i, 1}^{2}+\mathrm{Amp}_{i, 2}^{2}}, i \in[1, \ldots, 197]$

where $\mathrm{Amp}_{i}$ is the total amplitude of the 10-Hz Fourier component at dipole location $i$ and $\mathrm{Amp}_{i, 1}$ is the corresponding amplitude for the first and $\mathrm{Amp}_{i, 2}$ the amplitude for the second orientation.

\section{Statistical analysis}

Statistical analyses was executed in multiple steps. First, to assess an overall affective arousal effect on the neuromagnetic response, we determined the mean amplitude of the $10-\mathrm{Hz}$ Fourier component of the four cycles across all sensors for each subject. The mean amplitude for each affective category (pleasant, unpleasant, and neutral) was submitted to a contrast analysis to test the hypothesis that high-arousing stimuli generate a stronger neuromagnetic response than low-arousing pictures. Second, to evaluate broadly distributed effects of affective arousal on SSVEF sources, the 197 dipoles on the shell were grouped into six regional means for each hemisphere, resulting in occipital inferior, occipital superior, lateral inferior, lateral superior, frontal inferior, and frontal superior regions for the left and right hemisphere, respectively (see Fig. 1).

The mean amplitudes of these 12 regions were submitted to a repeated-measures analysis of variance (ANOVA) with factors of picture content (three levels: pleasant, neutral, unpleasant), hemisphere (two levels: left, right), and region (six levels: posterior inferior, posterior superior, lateral inferior, lateral superior, frontal inferior, and frontal superior). Main effects were tested by contrast analyses for a quadratic trend to test the hypothesis that high-arousing pleasant and unpleasant pictures produce a stronger brain response than low-arousing neutral pictures.

Pooling dipoles of the minimum norm shell to form regions for statistical analysis considerably reduces the spatial information gained by using 197 dipoles. Therefore, in a second step, a contrast analysis for a quadratic trend was performed for each of the 197 dipoles and the $F$ values were mapped on the same shell as used by the MNE procedure. This visualization of the results provides higher spatial resolution and specificity and was intended to complement the repeated-measures ANOVA described above. 


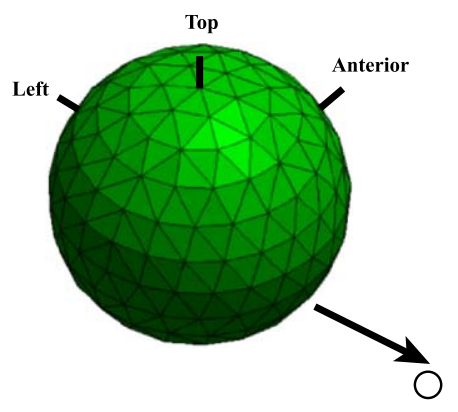

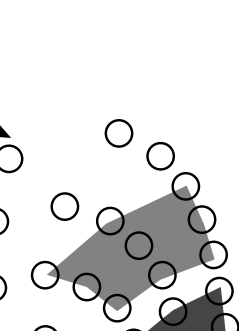

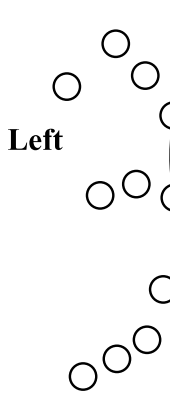

○

$\bigcirc$

Anterior

$\stackrel{\circ}{\bigcirc}$<smiles>[CH]OC[C@H](C)O</smiles>

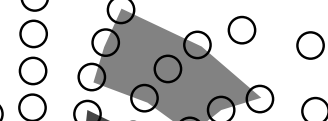

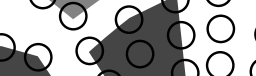

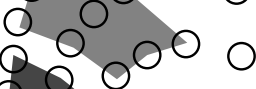

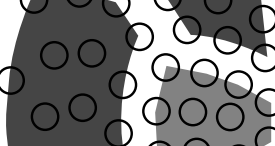

(1)

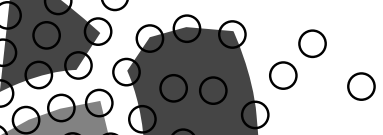

0
0
0
0
0
0
00000000000000000

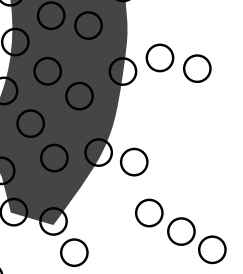

○<smiles></smiles>

O<smiles>[O]</smiles><smiles>[O-]</smiles><smiles>[O]C1CCCO1</smiles><smiles>[O-]</smiles>

Fig. 1. The upper left shell shows the triangulated MNE sphere, each node representing one of 197 dipole locations. On the bottom right, the 197 dipole sites are projected onto a plain. Gray shadings depict the six regions of interest for each hemisphere (occipital inferior, occipital superior, lateral inferior, lateral superior, frontal inferior, and frontal superior).

The HR data were scored according to a triphasic model of HR change over time (Hodes et al., 1985). The minimum bpm value in the first $2 \mathrm{~s}$ after stimulus onset was scored as the initial deceler- ation, the maximum value in the following $2 \mathrm{~s}$ was scored as the following acceleration, and the minimum in the last $2 \mathrm{~s}$ were scored as the final deceleration.

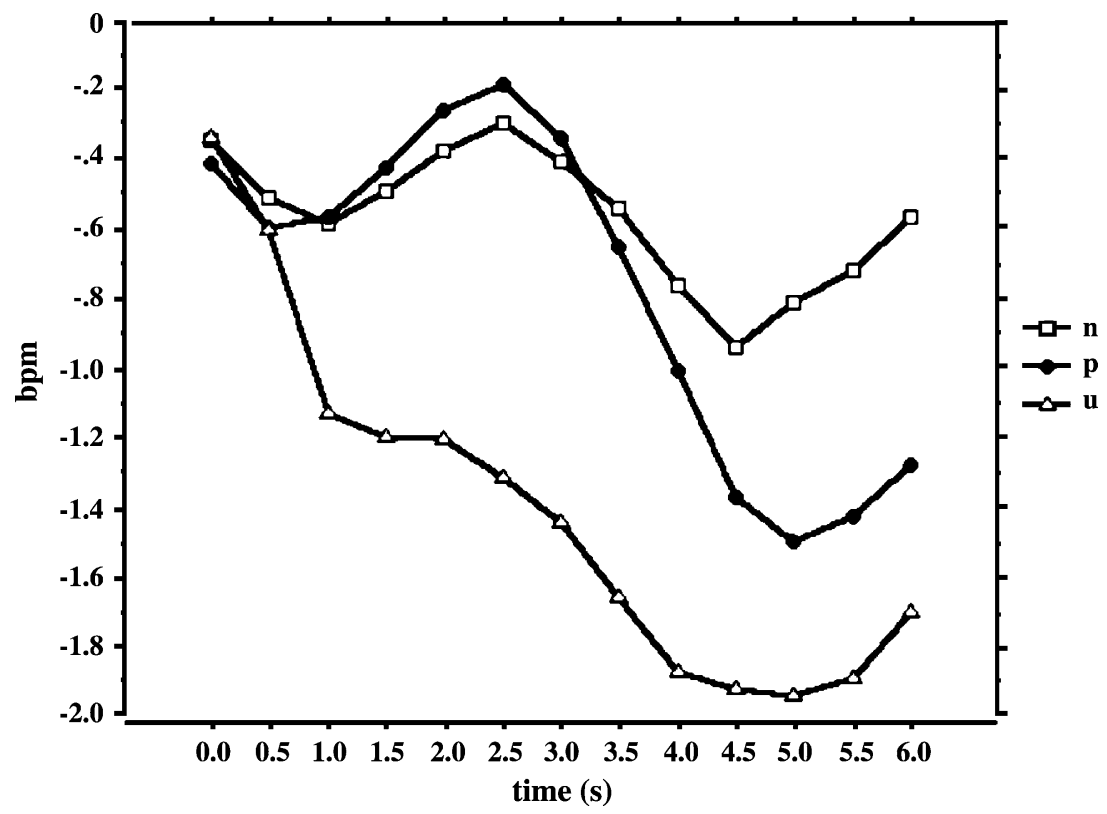

Fig. 2. Mean heart rate (HR) change relative to pre-stimulus baseline $(2 \mathrm{~s})$ across picture presentation time $(6 \mathrm{~s})$ for each affective category: $\mathrm{n}=\mathrm{neutral}, \mathrm{p}=$ pleasant, $\mathrm{u}=$ unpleasant. The overall pattern for all categories followed a triphasic pattern $[F(2,28)=13.37, P<0.001, \varepsilon=0.79]$, whereas unpleasant pictures elicited the greatest HR deceleration $[F(2,28)=9.03, P<0.01, \varepsilon=0.93]$. 

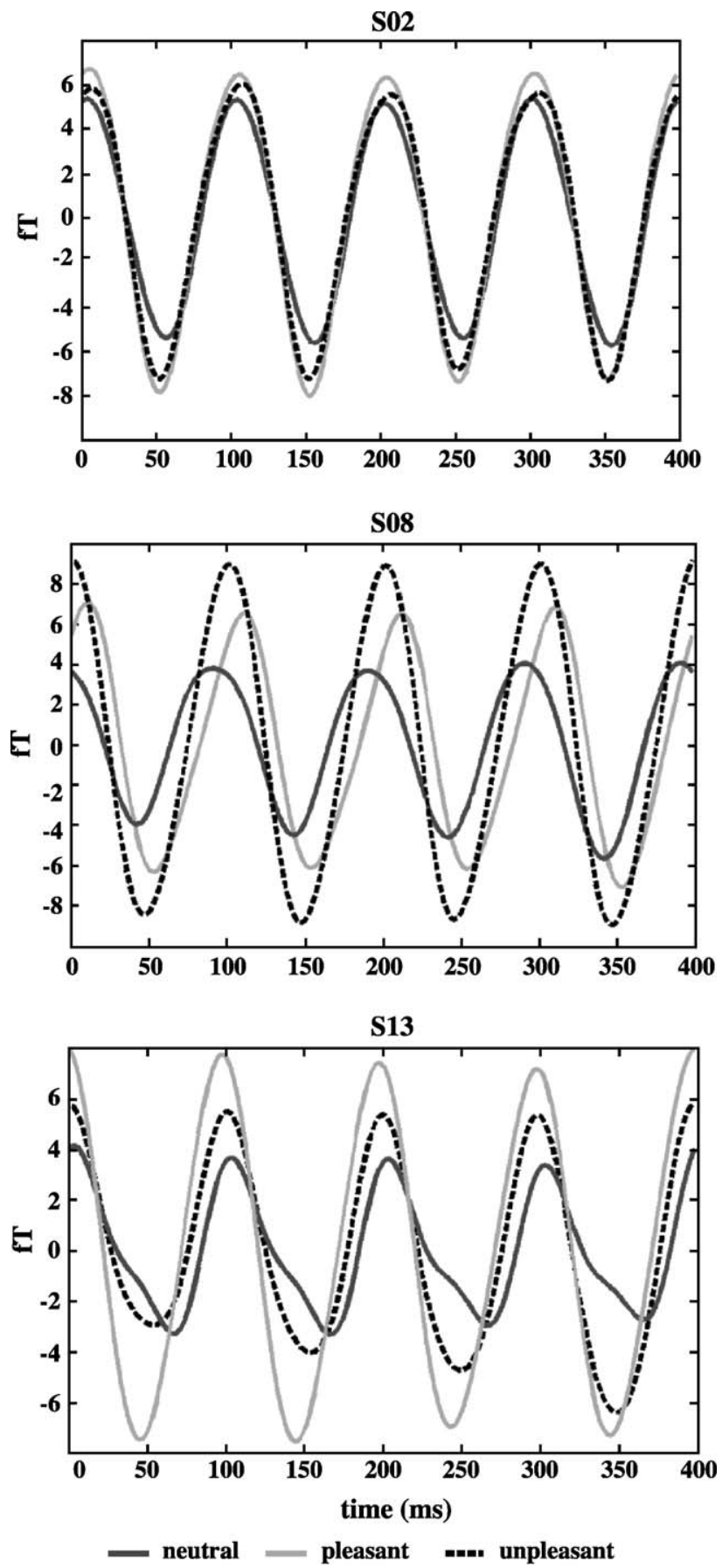

Fig. 3. Four cycles of the 10-Hz SSVEF response of a MEG sensor for three representative subjects. Shadings of curves indicate the SSVEFs elicited by neutral, pleasant, and unpleasant affective pictures.

Viewing times below or above two standard deviations of the mean within one affective category were rejected for each subject before calculating the average viewing time. For each subject, viewing times for each condition were divided by the mean viewing time over all three affective categories (pleasant, neutral, unpleasant) for normalization. HR data and viewing times were evaluated by repeated-measures ANOVA with factor emotional content (three levels: unpleasant, neutral, unpleasant). In the analysis of HR data, a factor phase (three levels: initial deceleration, following acceleration, final deceleration) was added. In all ANOVA analyses, the degrees of freedom were corrected where appropriate using the Greenhouse and Geisser (1959) procedure to account for possible violations of the sphericity assumption. Planned comparisons (contrast analyses) were used to examine the hypothesized differences related to affective valence and arousal. Where no planned comparisons were conducted, the Tukey-Kramer procedure and the $t$ test were utilized to evaluate main effects and interactions. SAM pleasure and arousal ratings were evaluated using repeatedmeasures ANOVA with a single factor of picture content (pleasant, neutral, unpleasant).

\section{Results}

\section{SAM ratings}

As expected, SAM pleasure ratings differed as a function of affective category $[F(2,28)=40.32, P<0.0001, \varepsilon=.55]$, with pleasant pictures rated as most pleasant followed by neutral pictures, and unpleasant pictures rated as least pleasant [mean pleasure ratings: $6.58 \pm 0.33$ (pleasant), $5.01 \pm 0.12$ (neutral), $2.64 \pm 0.30$ (unpleasant), Tukey-Kramer $P<0.05$ for all comparisons]. Arousal ratings also varied over affective categories $[F(2,28)=48.67, P<0.0001, \varepsilon=0.85]$, with pleasant and unpleasant pictures rated as more arousing than neutral pictures [mean arousal ratings: $4.80 \pm 0.33$ (pleasant), $2.59 \pm 0.31$ (neutral), $6.21 \pm 0.32$ (unpleasant), Tukey-Kramer $P<0.05$ for all comparisons]. Unpleasant pictures were rated as more arousing than pleasant pictures $[t(14)=4.9, P<0.001]$, which was consistent with the IAPS normative ratings.

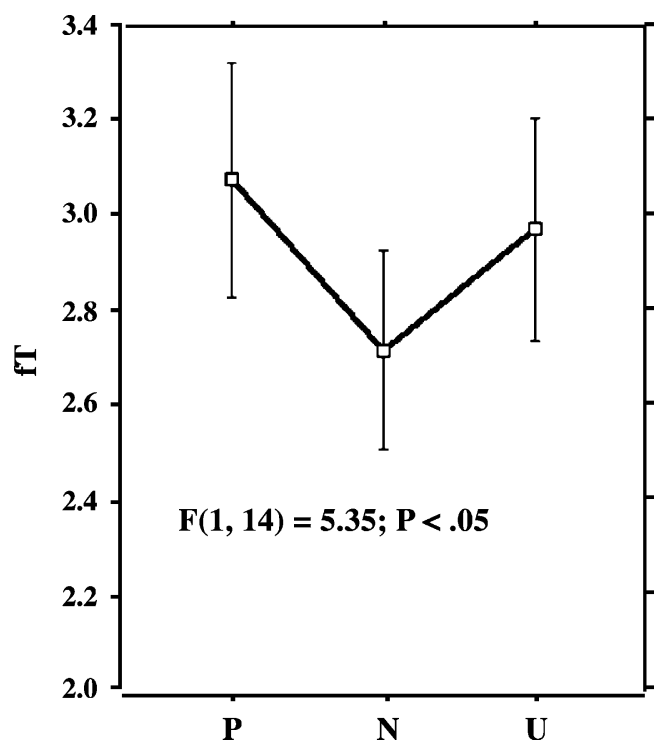

Fig. 4. Mean amplitude estimate of the $10-\mathrm{Hz}$ Fourier component of the SSVEF over all sensors and for each affective category: $\mathrm{P}=$ pleasant, $\mathrm{N}=$ neutral, $\mathrm{U}=$ unpleasant. High-arousing unpleasant and pleasant pictures generated a higher SSVEF response than low-arousing neutral pictures $[F(1,14)=5.35, P<0.05]$. Note that the high standard errors are due to the inter-subject variability of amplitude not affecting the significance of the within subject comparison. 


\section{Heart rate}

As hypothesized, the first, the following, and the last $2 \mathrm{~s}$ of heart rate changes during the 6-s picture presentation differed according to a triphasic model for all affective categories as detected by a main effect of phase $[F(2,28)=13.37, P<0.001, \varepsilon$ $=0.79$ (see Fig. 2)]. This difference was characterized by an initial deceleration, a following relative acceleration, and a final deceleration [mean initial deceleration: $-1.024 \pm 0.17$, mean following acceleration: $-0.477 \pm 0.21$, mean final deceleration: $-1.53 \pm 0.23$, Tukey-Kramer $P<0.05$ for all comparisons]. There was no interaction between affective category and phase of heart rate change.

However, a main effect of affective category $[F(2,28)=9.03$, $P<0.01, \varepsilon=0.93]$ revealed that the triphasic pattern of heart rate change elicited by unpleasant pictures was dominated by a stronger deceleration than the heart rate change elicited by neutral and pleasant pictures [mean deceleration: $-1.61 \pm 0.20$ (unpleasant), $-0.60 \pm 0.19$ (neutral), $-0.84 \pm 0.23$ (pleasant), Tukey-Kramer $P<0.05$ for comparisons between unpleasant, neutral and unpleasant, pleasant pictures]. There was no difference between neutral and pleasant pictures. The mean HR change pattern over subjects for each affective category is depicted in Fig. 2.

Viewing time

As expected, viewing times were longer for high-arousing pleasant and unpleasant pictures than for low-arousing neutral pictures, resulting in a significant main effect $[F(2,28)=7.55$, $P<0.005, \varepsilon=0.94]$ and a quadratic trend $[F(1,14)=17.28$, $P<0.005]$.

\section{SSVEF data}

Four cycles of the SSVEF response measured from three representative subjects at one (A42, parietal location) of the 148 sensors are shown in Fig. 3. The overall activation for each affective category as reflected by the mean amplitude estimate of the $10-\mathrm{Hz}$ Fourier component of the four cycles over all sensors of the extracted SSVEF signal differed according to a quadratic trend $[F(1,14)=5.35, P<0.05]$. The overall neuromagnetic activity of the signal space was higher for high-arousing pleasant and unpleasant stimuli than for calm neutral pictures (Fig. 4).
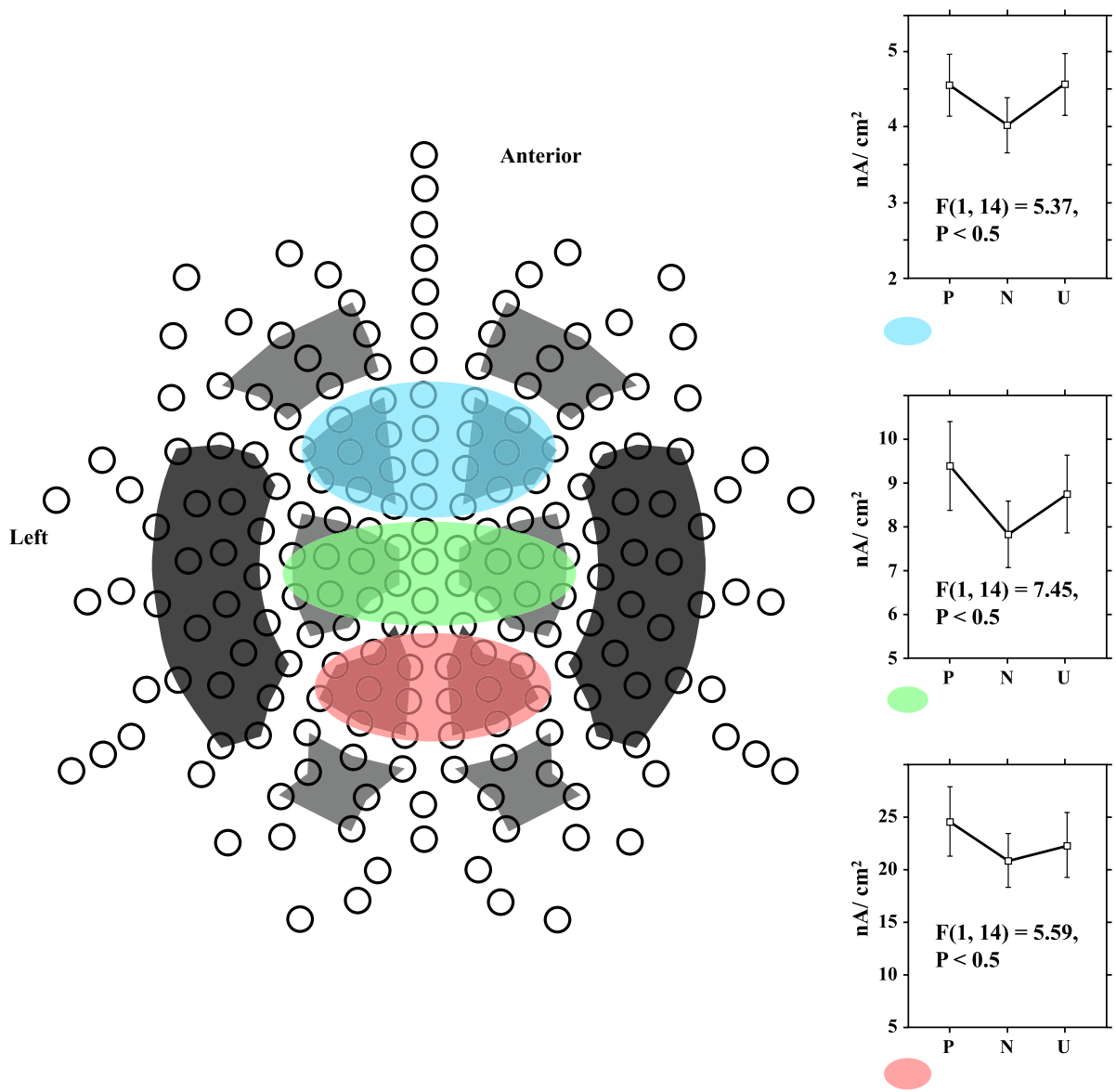

Fig. 5. Regions of interest depicting a significant arousal modulation of dipole source strength are shown on the right. The blue area represents superior frontal regions [arousal modulation: $F(1,14)=5.37, P<0.05$ ], the green area depicts superior lateral regions [arousal modulation: $F(1,14)=7.45, P<0.05$ ] and the red area shows superior posterior regions [arousal modulation: $F(1,14)=5.59, P<0.05$ ]. Right panels: mean amplitude of dipole source strength of the 10-Hz SSVEF for each affective category and each region of interest, which proved to be significant (color dot): $\mathrm{P}=$ pleasant, $\mathrm{N}=$ neutral, $\mathrm{U}=$ unpleasant. Note that the high standard errors are due to the high inter-subjects variability (different regularization parameters for each subject) not affecting the significance of the within-subject comparison. 


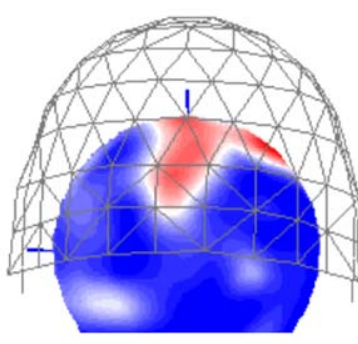

back view

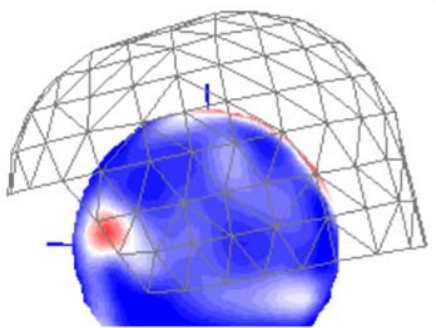

left view

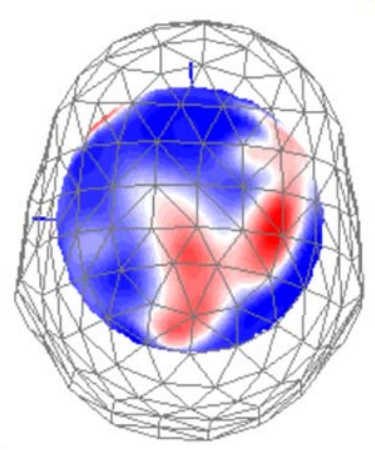

top view

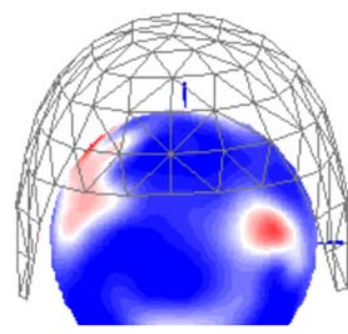

front view

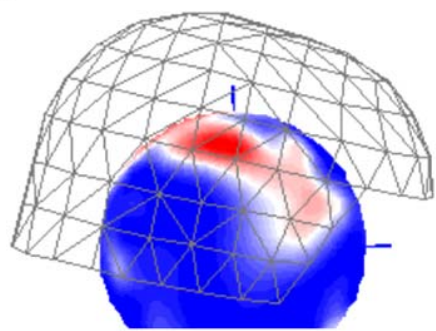

right view

\section{F-Value}

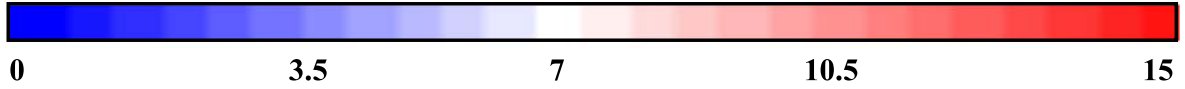

Fig. 6. Mapping of statistical parameters testing the hypothesis that high-arousing stimuli (pleasant and unpleasant vs. neutral) generate greater dipole strength. The color bar indicates the $F$ value level. The different panels depict different views of the MNE shell containing the dipole locations the $F$ values are projected on. $F$ values lower than 7 are shown in blue and values greater than 7 are depicted in red (see text). The gray net represents the mean MEG sensor positions (each node represents one sensor) for the 15 subjects.

\section{Minimum norm estimate}

To get information about the origin of this SSVEF modulation, the mean $10-\mathrm{Hz}$ Fourier component amplitude estimates of 12 dipole clusters as shown in Fig. 1 were submitted to statistical

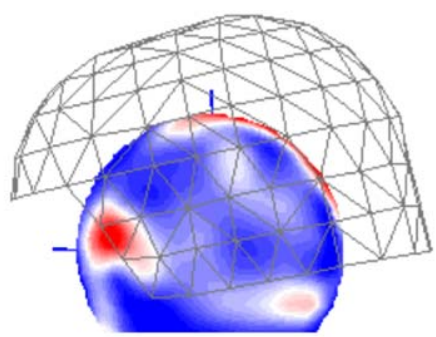

left view analysis. A main effect of region $[F(5,70)=35.15 ; P<0.0001$; $\varepsilon=0.32]$ indicated that the visual stimulation elicited most activity over inferior and superior posterior regions as compared to all other areas [Tukey-Kramer $P<0.05$ for all comparisons between the two posterior and all other regions; the comparison between inferior and

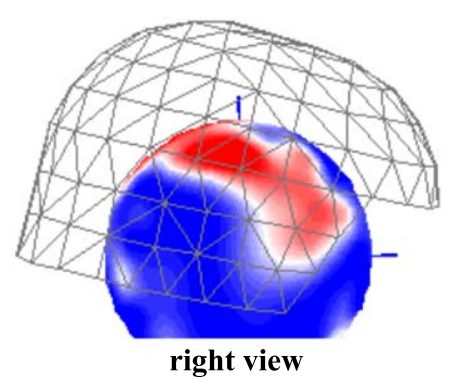

F-Value

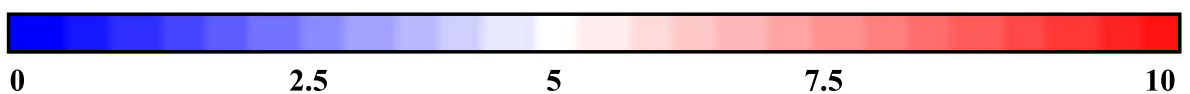

Fig. 7. Left and right view of the statistical parameter map of Fig. 6. The scale of the $F$ map was downscaled to depict weaker activity modulations. As can be seen, arousal modulations also emerge in the ventral visual stream. The gray net represents the mean MEG sensor positions (each node represents one sensor) for the 15 subjects. 
superior posterior regions did not reach significance]. A significant interaction of region and affective category $[F(10,140)=$ $3.52, P<0.05, \varepsilon=0.30]$ revealed that high-arousing pictures (pleasant and unpleasant) also generated more activity than low-arousing pictures (neutral) in frontal superior $[F(1,14)=5.37, P<0.05]$, lateral superior $[F(1,14)=7.45, P<0.05]$ and occipital superior $[F(1,14)=5.59, P<0.05]$ regions (Fig. 5).

Averaging activity clusters of dipoles reduces spatial resolution and leads to loss of information. A contrast analysis for a quadratic trend testing the hypothesis that high-arousing pictures produce more activity than calm neutral pictures was therefore conducted for all 197 dipoles of the minimum norm shell. This resulted in a descriptive $F$ map (Fig. 6).

$F$ values greater than $7[F(1,14)=7.0 ; P=0.007$, uncorrected] are shown in red color and $F$ values less than 7 are depicted in blue. As can be seen in Fig. 6, $F$ values greater than 7 distribute across frontoparietal regions with a predominance on the right hemisphere. The maximum $F$ value is located at a right parietal dipole site $[F(1,14)=15.1]$ as can be seen in the middle panel of Fig. 6 (top view). An additional cluster of high $F$ values is also found in left frontal regions [maximal $F$ value in that cluster: $F(1$, $14)=13.0]$. If the arbitrary threshold for the red color in Fig. 6 is lowered to an $F$ value of $5[F(1,14)=5.0]$ to depict weaker modulations, high-arousing affective pictures also show greater activity than neutral pictures in occipito-temporal regions [maximum of $F$ value in right occipito-temporal cluster: $F(1,14)=4.79$; maximum of $F$ value in left occipito-temporal cluster: $F(1,14)=$ 6.14; see Fig. 7] with a left-hemispheric predominance.

\section{Discussion}

Examining MEG correlates of affective picture viewing by steady state visual evoked fields, we studied the modulation of stimulus-driven neural responses to visual stimuli differing as to their affective/motivational significance for the observer. The overall SSVEF response as reflected by the mean amplitude of the 10-Hz Fourier component over all MEG sensors suggested that high-arousing pictures (pleasant and unpleasant) produced a greater SSVEF response than low-arousing neutral pictures. This finding is consistent with studies using other central nervous measures of emotional picture processing reporting that indices of higher-order processing of a stimulus varied as a function of emotional arousal (Cuthbert et al., 2000; Ito et al., 1998; Mini et al., 1996; Palomba et al., 1997; Schupp et al., 2000).

In attention research, investigating visual spatial selective attention over time by SSVEPs revealed that the SSVEP amplitude is greater for attended than for unattended visual stimuli (Morgan et al., 1996; Muller and Hubner, 2002). This effect is understood as an early sensory gain mechanism that enhances the signal-to-noise ratio in the underlying cortical networks and hence facilitates discrimination of the attended visual stimulus (Hillyard and Anllo-Vento, 1998). We suggest that the greater SSVEF amplitude while viewing arousing pictures may reflect a sensory gain mechanism interacting with a frontoparietal attention mechanism regulating and maintaining the alert state (see below), reflecting 'automatic', motivated attention to biologically significant stimuli.

Assuming that steady state stimulation with emotional pictures leads to stimulus-driven activity in cortical networks underlying affective picture processing, we conducted sources analysis. Esti- mating the sources of the SSVEF by a distributed source analysis technique, we observed that averaged dipole cluster strengths at superior occipital, superior lateral, and superior frontal regions of interests were greater for high-arousing than for low-arousing pictures. This effect was greatest for superior lateral dipole clusters that were most sensitive to parietal cortical activity. Mapping of statistical parameters indicated that the modulation of source strength as a function of emotional arousal was greatest at frontoparietal locations, with a predominance at right hemispheric dipole sites. The greatest $F$ value was located at a right parietal dipole site, thus paralleling and confirming the dipole cluster analysis. Furthermore, a strong modulation by emotional arousal was observed at left frontal locations. Such a modulation was also evident at occipito-temporal dipole sites but to a much lesser extent. This pattern suggests that the SSVEF was greater for high-arousing than for low-arousing pictures in a frontoparietal cortical network showing a right hemisphere preponderance, and maybe interacting with changes in occipito-temporal cortex. The spatio-temporal relationship between these two networks will be a target of future research using SSVEF together with measures of interdipole phase-locking.

While SSVEP studies of spatial selective attention have not employed source estimation techniques, event-related potentials (ERP) studies in that field showed early modulations in extrastriate cortex (Hillyard and Anllo-Vento, 1998) and recurrent modulations in primary visual cortex (Di Russo et al., 2003). These results are paralleled by findings of early ERP modulations with affective arousal (Keil et al., 2002; Pizzagalli et al., 1999), leading us to the assumption that a similar sensory gain mechanism as has been suggested for the field of spatial attention could mediate the allocation of motivated attention.

We observed arousal-related modulations in extrastriate regions (at occipito-temporal dipole sites) in addition to pronounced changes at frontoparietal dipole locations. In our previous SSVEP study (Keil et al, in press) using the same picture material, we found a corresponding pattern of enhanced amplitudes at occipital and parietal electrode sites and coactivation of frontal and temporal electrode sites during presentation of high-arousing pictures. The topography of amplitude modulations of the present study at occipito-temporal and right parietal dipole sites is consistent with current source density maps reported by Junghöfer et al. (2001) who presented IAPS pictures in a rapid visual stream. Given that the SSVEF segment examined here reflects neuromagnetic activity over a $6000-\mathrm{ms}$ window, it might be expected that structures related to allocation/regulation of attention show a more sustained pattern than do their target structures in visual cortex.

Compared to other measures of brain activity like fMRI and PET, our source estimation results are in line with findings from attention research using metabolic imaging techniques. The modulation of activity at frontoparietal dipole sites by the arousal dimension of emotional pictures observed here seems to involve cortical networks also discussed in attention research (Corbetta, 1998; Le et al., 1998; Pardo et al., 1991; Rushworth et al., 2001) and parallels results about orienting and maintaining the alert state (Fernandez-Duque and Posner, 2001). Taken together, the enhancement of the SSVEF and the spatial distribution of the arousal modulated dipole strengths in our study suggests that motivationally significant visual stimuli may engage a sensory gain mechanism facilitating sensory processing in extrastriate cortex interacting with the activation of a common cortical attention network mediating orienting and maintaining the alert state over picture 
presentation time, supporting the concept of 'motivated attention' in emotion (Lang et al., 1997).

Because of the experimental environment (MEG chamber) and the application of a SSVEF design with flickering pictures, it is important to ensure that we have triggered the same physiological and behavioral responses usually observed in an affective picture viewing context. Viewing emotional pictures usually elicits a classic triphasic pattern of heart rate change (Lang and Hnatiow, 1962) with an initial deceleration, a subsequent relative acceleration, and a final deceleration. Aversive pictures generate a sustained heart rate deceleration over the whole picture presentation time with a less clear triphasic pattern compared to neutral and pleasant pictures (Lang et al., 1997). Pleasant pictures usually provoke a greater initial deceleration and a more pronounced accelerative component than neutral pictures (Bradley et al., 2001). In our study, we observed similar heart rate changes during affective picture viewing in the MEG chamber, although the pictures were presented in a flickering mode. High-arousing unpleasant stimuli produced a sustained heart rate deceleration over the whole picture presentation time. However, in the present study, we could not replicate a differentiation between pleasant and neutral pictures with respect to heart rate change. This could be due to the more aversive experimental environment of the MEG chamber. Another reason may lie in the high variability between subjects usually observed in heart rate data (Hodes et al., 1985). It is possible that with a small-sized sample (compared to sample sizes usually used in heart rate studies), only the most pronounced changes related to aversive pictures lead to significant effects, whereas smaller differences such as the ones between neutral and pleasant pictures require bigger samples. This would especially hold in situations where motivational significance is highest for aversive pictures across subjects. Indeed, aversive pictures were rated as slightly more arousing than pleasant images in the present study. Further, the flickering mode of picture presentation did not influence subjective ratings and viewing time usually observed (Lang et al., 1998). As predicted, subjects' ratings validated that the categorization of the IAPS pictures according to the normative ratings indeed represented high (pleasant and unpleasant)- and low (neutral)-arousing affective pictures. Furthermore, subjects viewed high-arousing pictures longer than neutral pictures during SAM rating sessions following the MEG recordings, possibly reflecting a higher motivational state to high-arousing pictures. Thus, behavioral and physiological data suggest that we successfully manipulated the motivational significance of our stimuli in a SSVEF paradigm. Modulation of SSVEF amplitude therefore appears to reflect changes related to emotional arousal as a function of picture category.

However, several methodological constraints must be taken into account. First, we focused on amplitude data of the 10-Hz Fourier component and dismissed the phase information. This was done as our main goal was to use source space projection, allowing for conclusions about the origin of neuromagnetic activity. Magnetometer locations of maximum activity do not represent the origin of the sources. Additionally, subjects have different relative head positions in the MEG sensor helmet. Hence, we employed a MNE algorithm (Hauk et al., 1998) whereby two dipole orientations at each model dipole site must be taken into account (see above). While meaningful amplitude information can easily be obtained as described in Methods, pooling phase information across two perpendicular dipoles leads to distortions, which makes interpre- tation difficult. As a second constraint, the present study employed a homogenous sphere as a head model for the MNE. Thus, cortical areas involved in generation of the signal were inferred from localizations on a shell. But we consider this approach more accurate than inferring activation of cortical areas from sensor/ electrode topographies because sensors/electrodes can be sensitive to distant cortical sources. In future studies, realistic head models derived from subjects' individual structural MRIs should be used to obtain more precise identification of brain regions.

The spatial distribution of the activity changes at the stimulusdriven SSVEF response suggests that whenever visual emotional stimuli engage attentional resources extrastriate and higher-order cortical attention networks also involved in orienting and maintaining the alert state (Fernandez-Duque and Posner, 2001) are active. We propose that an initial sensory gain mechanism as well as a general attention system mediating orienting and an alert state over time maintain an enhanced signal-to-noise ratio in these structures as reflected by the enhanced SSVEF neuromagnetic response. The higher-order attention network may interact with lower sensory systems processing stimulus features by top down processes. This view would be consistent with the theory of emotion proposed by Lang (1979), suggesting that affective information is integrated and represented in neuronal networks. The interplay of the above-mentioned mechanisms in time should be investigated by future research.

\section{Acknowledgments}

This research was supported by the Deutsche Forschungsgesellschaft (DFG) and the Volkswagenstiftung.

\section{References}

Berg, P., Scherg, M., 1994. A multiple source approach to the correction of eye artifacts. Electroencephalogr. Clin. Neurophysiol. 90, 229-241.

Bertero, M., De Mol, C., Pike, E.R., 1988. Linear inverse problems with discrete data II: stability and regularization. Inverse Probl. 4, 573-594.

Bradley, M.M., Codispoti, M., Cuthbert, B.N., Lang, P.J., 2001. Emotion and motivation I: defensive and appetitive reactions in picture processing. Emotion 1, 276-298.

Corbetta, M., 1998. Frontoparietal cortical networks for directing attention and the eye to visual locations: identical, independent, or overlapping neural systems? Proc. Natl. Acad. Sci. U. S. A. 95, 831-838.

Cuthbert, B.N., Schupp, H.T., Bradley, M.M., Birbaumer, N., Lang, P.J., 2000. Brain potentials in affective picture processing: covariation with autonomic arousal and affective report. Biol. Psychol. 52, 95-111.

CSEA (Center for the Study of Emotion and Attention), 1999. International Affective Picture System (IAPS): Technical Manual and Affective Ratings. NIMH-Center for the Study of Emotion and Attention. University of Florida, Gainesville, FL.

Davidson, R.J., Abercrombie, H., Nitschke, J.B., Putnam, K., 1999. Regional brain function, emotion and disorders of emotion. Curr. Opin. Neurobiol. 9, 228-234.

Di Russo, F., Martinez, A., Hillyard, S.A., 2003. Source analysis of eventrelated cortical activity during visuo-spatial attention. Cereb. Cortex 13, 486-499.

Fernandez-Duque, D., Posner, M.I., 2001. Brain imaging of attentional networks in normal and pathological states. J. Clin. Exp. Neuropsychol. 23, 74-93.

Grave de Peralta Menendez, R., Hauk, O., Gonzalez Andino, S., Vogt, H., Michel, C., 1997. Linear inverse solutions with optimal resolution ker- 
nels applied to electromagnetic tomography. Hum. Brain Mapp. 5, $454-467$.

Greenhouse, S.W., Geisser, S., 1959. On methods in the analysis of profile data. Psychometrika 24, 95-112.

Hämäläinen, M.S., Ilmoniemi, R.J., 1994. Interpreting measured magnetic fields of the brain: minimum norm estimates. Med. Biol. Eng. Comput. $32,35-42$.

Hauk, O., Berg, P., Wienbruch, C., Rockstroh, B., Elbert, T., 1998. The minimum norm method as an effective mapping tool for MEG analysis. Biomag98, Sendai, Japan.

Hauk, O., Keil, A., Elbert, T., Müller, M.M., 2002. Comparison of data transformation procedures to enhance topographical accuracy in time series analysis of the human EEG. J. Neurosci. Methods $113,111-122$.

Heller, W., Nitschke, J.B., Etienne, M.A., Miller, G.A., 1997. Patterns of regional brain activity differentiate types of anxiety. J. Abnorm. Psychol. 106, 376-385.

Hillyard, S.A., Anllo-Vento, L., 1998. Event-related brain potentials in the study of visual selective attention. Proc. Natl. Acad. Sci. U. S. A. 95, $781-787$

Hodes, R.L., Cook III, E.W., Lang, P.J., 1985. Individual differences in autonomic response: conditioned association or conditioned fear? Psychophysiology $22,545-560$.

Ito, T.A., Larsen, J.T., Smith, N.K., Cacioppo, J.T., 1998. Negative information weighs more heavily on the brain: the negativity bias in evaluative categorizations. J. Pers. Soc. Psychol. 75, 887-900.

Junghöfer, M., Bradley, M.M., Elbert, T.R., Lan, P.J., 2001. Fleeting images: a new look at early emotion discrimination. Psychophysiology 38 , $175-178$.

Keil, A., Bradley, M.M., Hauk, O., Rockstroh, B., Elbert, T., Lang, P.J., 2002. Large-scale neural correlates of affective picture processing. Psychophysiology 39, 641-649.

Keil A., Gruber T., Müller M.M., Moratti S., Stolarova M., Bradley M.M., Lang P.J., 2003. Early modulation of visual perception by emotional arousal: evidence from steady-state visual evoked brain potentials. Cogn. Affect. Behav. Neurosci. 3, 195-206.

Kemp, A.H., Gray, M.A., Eide, P., Silberstein, R.B., Nathan, P.J., 2002. Steady-state visually evoked potential topography during processing of emotional valence in healthy subjects. NeuroImage 17, 1684-1692.

Knosche, T., Praamstra, P., Stegeman, D., Peters, M., 1996. Linear estimation discriminates midline sources and a motor cortex contribution to the readiness potential. Electroencephalogr. Clin. Neurophysiol. 99, $183-190$

Lang, P.J., 1979. A bioinformational theory of emotional imagery. Psychophysiology $16,495-512$.

Lang, P.J., 1980. Behavioral treatment and bio-behavioral assessment: computer applications. In: Sidowski, J.B., Johnson, J.H., Williams, T.A. (Eds.), Technology in Mental Health Care Delivery Systems. Ablex Publishing, Norwood, NJ, pp. 119-137.

Lang, P.J., Hnatiow, M., 1962. Stimulus repetition and the heart rate response. J. Comp. Physiol. Psychol. 55, 781-785.

Lang, P.J., Bradley, M.M., Cuthbert, B.N., 1997. Motivated attention: affect, activation, and action. In: Lang, P.J., Simons, R.F., Balaban, M.T. (Eds.), Attention and Orienting: Sensory and Motivational Processes. Lawrence Erlbaum Associates, Hillsdale, NJ, pp. 97-135.

Lang, P.J., Bradley, M.M., Cuthbert, B.N., 1998. Emotion, motivation, and anxiety: brain mechanisms and psychophysiology. Biol. Psychiatry 44, $1248-1263$.
Le, T.H., Pardo, J.V., Hu, X., 1998. 4 T-fMRI study of nonspatial shifting of selective attention: cerebellar and parietal contributions. J. Neurophysiol. 79, 1535-1548.

LeDoux, J.E., 1992. Emotion and the amygdala. In: John, P.A. (Ed.), The Amygdala: Neurobiological Aspects Of Emotion, Memory, and Mental Dysfunction. Wiley-Liss, New York, NY, pp. 339-351.

Mini, A., Palomba, D., Angrilli, A., Bravi, S., 1996. Emotional information processing and visual evoked brain potentials. Percept. Mot. Skills 83, $143-152$.

Morgan, S.T., Hansen, J.C., Hillyard, S.A., 1996. Selective attention to stimulus location modulates the steady-state visual evoked potential. Proc. Natl. Acad. Sci. U. S. A. 93, 4770-4774.

Müller, M.M., Hillyard, S., 2000. Concurrent recording of steady-state and transient event-related potentials as indices of visual-spatial selective attention. Clin. Neurophysiol. 111, 1544-1552.

Müller, M.M., Teder-Salejarvi, W., Hillyard, S.A., 1998. The time course of cortical facilitation during cued shifts of spatial attention. Nat. Neurosci. $1,631-634$.

Muller, M.M., Hubner, R., 2002. Can the spotlight of attention be shaped like a doughnut? Evidence from steady-state visual evoked potentials. Psychol. Sci. 13, 119-124.

Oldfield, R.C., 1971. The assessment and analysis of handedness: the Edinbourgh Inventory. Neuropsychologia 9, 97-113.

Palomba, D., Angrilli, A., Mini, A., 1997. Visual evoked potentials, heart rate responses and memory to emotional pictorial stimuli. Int. J. Psychophysiol. 27, 55-67.

Pardo, J.V., Fox, P.T., Raichle, M.E., 1991. Localization of a human system for sustained attention by positron emission tomography. Nature 349 , $61-64$.

Perlstein, W.M., Cole, M.A., Larson, M., Kelly, K., Seignourel, P., Keil, A., 2003. Steady-state visual evoked potentials reveal frontally-mediated working memory activity in humans. Neurosci. Lett. 342, 191-195.

Picton, T.W., Vajsar, J., Rodriguez, R., Campbell, K.B., 1987. Reliability estimates for steady-state evoked potentials. Electroencephalogr. Clin. Neurophysiol. 68, 119-131.

Pizzagalli, D., Regard, M., Lehmann, D., 1999. Rapid emotional face processing in the human right and left brain hemispheres: an ERP study. NeuroReport 10, 2691-2698.

Plourde, G., Picton, T.W., 1990. Human auditory steady-state response during general anesthesia. Anesth. Analg. 71, 460-468.

Regan, D., 1989. Human brain electrophysiology: evoked potentials and evoked magnetic fields in science and medicine. Elsevier, New York.

Rushworth, M.F., Paus, T., Sipila, P.K., 2001. Attention systems and the organization of the human parietal cortex. J. Neurosci. 21, 5262-5271.

Schupp, H.T., Cuthbert, B.N., Bradley, M.M., Cacioppo, J.T., Ito, T., Lang, P.J., 2000. Affective picture processing: the late positive potential is modulated by motivational relevance. Psychophysiology 37 , $257-261$.

Shi, C., Davis, M., 2001. Visual pathways involved in fear conditioning measured with fear-potentiated startle: behavioral and anatomic studies. J. Neurosci. 21, 9844-9855.

Silberstein, R.B., Schier, M.A., Pipingas, A., Ciorciari, J., Wood, S.R., Simpson, D.G., 1990. Steady-state visually evoked potential topography associated with a visual vigilance task. Brain Topogr. 3, $337-347$.

Srinivasan, R., Russell, D.P., Edelman, G.M., Tononi, G., 1999. Increased synchronization of neuromagnetic responses during conscious perception. J. Neurosci. 19, 5435-5448. 